Unfallchirurg 2016 $\cdot 119: 408-413$

DOI 10.1007/s00113-016-0174-4

Online publiziert: 9 . Mai 2016

(C) The Author(s) 2016. This article is available at SpringerLink with Open Access

\section{Redaktion}

C. Krettek, Hannover

C. Willy, Berlin

CrossMark

\author{
S. Salminger ${ }^{1,2} \cdot$ J. A. Mayer ${ }^{2} \cdot$ A. Sturma ${ }^{2,3} \cdot$ O. Riedl ${ }^{1,2} \cdot$ K. D. Bergmeister ${ }^{2} \cdot$ \\ O. C. Aszmann ${ }^{1,2}$ \\ ${ }^{1}$ Klinische Abteilung für Plastische und Rekonstruktive Chirurgie, Universitätsklinik für Chirurgie, \\ Medizinische Universität Wien, Wien, Österreich \\ ${ }^{2}$ Christian Doppler Labor für Wiederherstellung von Extremitätenfunktionen, Medizinische Universität \\ Wien, Wien, Österreich \\ ${ }^{3}$ Universitätsklinik für Physikalische Medizin und Rehabilitation, Medizinische Universität Wien, Wien, \\ Österreich
}

\title{
Prothetische Rekonstruktion der oberen Extremität
}

Erweiterung des Spektrums zur Wiederherstellung von Extremitätenfunktionen bieten [3].

Durch die technische Weiterentwicklung auf dem Gebiet der Prothetik und die dadurch verbesserte Akzeptanz von myoelektrischen Prothesen steigen auch die Anforderungen an den Patienten, den Stumpf und letztendlich die Funktionalität [14]. Die größten Fortschritte der letzten Jahre betrafen allerdings die Steuerungsmöglichkeiten und somit die Schnittstelle Mensch-Maschine [1, 13, 14].

In der folgenden Arbeit sollen die Möglichkeiten der prothetischen Rekonstruktion bei hohen Amputationen, aber auch bei erhaltener, jedoch funktionsloser Extremität dargestellt werden.

\section{Die hohe Amputation}

Konventionelle myoelektrische Prothesen bei transhumeralen und glenohumeralen Amputationen steuert man mit zwei Muskelsignalen an, welche von zwei getrennt innervierten Muskelgruppen der verbliebenen Stumpfmuskulatur stammen. Auf transhumeralem Amputationsniveau werden der $\mathrm{M}$. biceps und M. triceps, bei glenohumeralen Stümpfen meist der M. pectoralis major und der M. latissimus dorsi dafür verwendet. Die verschiedenen prothetischen Gelenke und Funktionen (Hand, Handgelenk, Ellenbogen) müssen mittels Kokontraktionen der erwähnten zwei Muskeln angewählt und in der jeweiligen Ebene mit denselben Muskeln linear angesteuert werden. Hierbei besteht die Schwierigkeit darin, dass ein und dieselbe kognitive „Bewegung“ mit verschiedenen prothetischen Funktionen belegt und somit für den Patienten nur unter größtmöglicher Konzentration einsetzbar ist. Ein harmonischer, dem natürlichen Bewegungsmuster einigermaßen entsprechender Bewegungsablauf ist mit diesem Steuerungsmechanismus nicht möglich, da eine simultane Bewegung mehrerer prothetischer Gelenke nicht durchführbar ist.

\section{Selektive Nerventransfers - Targeted Muscle Reinnervation}

Die chirurgische Multiplikation von Muskelsignalen zur Verbesserung der Prothesensteuerung beschrieben erstmals Kuiken et al. [8]. Hierbei werden die Nerven, die durch die Amputation ihr distales Zielorgan verloren haben, im Wesentlichen N. radialis, N. medianus, $\mathrm{N}$. ulnaris und bei glenohumeraler Amputation auch der N. musculocutaneus, an einzelne Muskeln des Stumpfes transferiert, um ebendort eine suffiziente Anzahl intuitiv steuerbarer Muskelsignale zu kreieren. Diese Technik der selektiven Nerventransfers bei Amputierten nennt man Targeted Muscle Reinnervation (TMR). Die Zielmuskeln spannen sich schließlich entsprechend der Aktivität der Spendernerven an und ihre 
Tab. 1 Nerventransfermatrix transhumerale Amputation

\begin{tabular}{lll}
\hline Zielmuskeln/Signalgeber & Nerv & Prothesenfunktion \\
\hline M. biceps caput longum & N. musculocutaneus & Ellenbogenflexion \\
\hline M. biceps caput breve & N. ulnaris & Hand Schließen \\
\hline M. brachialis & N. medianus & Pronation \\
\hline M. triceps caput longum/mediale & N. radialis & Ellenbogenextension \\
\hline M. triceps caput laterale & Geteilter Ramus prof. N. radialis & Hand Öffnen \\
\hline M. brachioradialis & Geteilter Ramus prof. N. radialis & Supination \\
\hline
\end{tabular}

Tab. 2 Nerventransfermatrix glenohumerale Amputation

\begin{tabular}{|lll|}
\hline Zielmuskeln/Signalgeber & Nerv & Prothesenfunktion \\
\hline M. pectoralis major Pars clavicularis & N. musculocutaneus & Ellenbogenflexion \\
\hline M. pectoralis minor & N. ulnaris & Hand Schließen \\
\hline M. pectoralis major Pars sternocostalis & N. medianus & Umwendbewegung \\
\hline M. pectoralis major Pars abdominalis & N. medianus & Umwendbewegung \\
\hline M. latissimus dorsi & N. radialis & Ellenbogenextension \\
\hline M. infraspinatus & Ramus prof. N. radialis & Hand Öffnen \\
\hline
\end{tabular}

Muskelpotenziale werden über Oberflächenelektroden an die Prothese weitergegeben. Ziel dieser Operation ist es, den Patienten mit bis zu sechs individuellen Muskelsignalen auszustatten. Auf diese Weise ist eine harmonische, intuitive, dem natürlichen Bewegungsmuster entsprechende Steuerung gewährleistet, ohne dass der Patient zwischen den verschiedenen Steuerungsebenen wechseln muss. Diese beträchtliche Erweiterung der Steuerungsmöglichkeiten führt $\mathrm{zu}$ einer deutlich verbesserten Funktionalität und somit zu einer deutlich erhöhten Patientenzufriedenheit und Akzeptanz der Prothese.

\section{》) Voraussetzung sind intakte Muskeln im Bereich des Amputationsstumpfes}

Voraussetzung für den Erfolg einer solchen Operation sind intakte Muskeln im Bereich des Amputationsstumpfes sowie ein weitgehend intaktes proximales Armnervengeflecht (Plexus brachialis) mit der Möglichkeit, Spendernerven entsprechend topografisch-anatomisch isolieren zu können. Diesbezüglich ist eine präoperative Magnetresonanztomographie (MRT), hochauflösender Ultraschall sowie bilanzierende Nervenleitgeschwindigkeit (NLG) und Elektromyographie (EMG) des verbliebenen Armnerven- geflechtes zu empfehlen. Weiters muss der Patient kognitiv dazu in der Lage sein, die verschiedenen Funktionen seines Phantomarmes $\mathrm{zu}$ visualisieren, und gewillt sein, ein intensives und manchmal auch langwieriges Signalund Prothesentraining $\mathrm{zu}$ absolvieren. Da die transferierten Nerven erst in ihre Zielmuskeln einwachsen müssen und das Signaltraining ebenfalls Zeit in Anspruch nimmt, ist mit einer abgeschlossenen TMR-Prothesenversorgung erst etwa 12 Monate nach Operation zu rechnen.

\section{Operative Technik}

Das Ziel der TMR-Operation ist es, die einzelnen Nerven des Plexus brachialis aus dem verbliebenen Stumpf bzw. der bestehenden Amputationsnarbe herauszulösen und an vorhandene neuromuskuläre Einheiten anzuschließen. Um möglichst gute topografische Trennung der einzelnen Muskeln notwendig. Die Operation erfolgt in Rückenlage des Patienten mit erhöhtem Oberkörper, der Kopf wird leicht zur Gegenseite gedreht. Je nach Unfallhergang beginnt die Operation mit einer infra- bzw. supraklavikulären Exploration des Plexus brachialis. Dies ist vor allem nach Schleudertraumata bzw. Traktionsschäden des Plexus zu empfehlen, um etwaige proximale Nerdie Signalqualität zu optimieren, ist eine venschäden auszuschließen und wenn notwendig $\mathrm{zu}$ behandeln. Infolge werden die Nervenäste der Zielmuskulatur dargestellt. Diese unterscheiden sich je nach Amputationshöhe.

Bei transhumeral Amputierten werden die einzelnen Spendernerven des Plexus brachialis in der Regel an die Muskeläste des M. biceps caput breve, M. brachialis, M.tricepscaputlaterale und wenn vorhanden des M. brachioradialis transferiert. Im Anschluss an die Identifizierung aller Zielmuskeln wird der kurze Kopf des M. biceps von seinem Ansatz am Coracoid gelöst und nach medial abgedrängt, um eine bessere räumliche Trennung zu erreichen. Nach erfolgter mikrochirurgischer Präparation bzw. nach Aufsuchen und Anschlingen der Neurome von N. medianus, N. ulnaris und Ramus prof. N. radialis sowie nach Freilegung der einzelnen Muskeläste von $\mathrm{N}$. musculocutaneus und N. radialis begutachtet man die Situation erneut und erstellt eine sog. Nerventransfermatrix anhand der anatomischen Gegebenheit. Trotz auswärts durchgeführter Neuromrückkürzungen waren aufgrund dieser individuellen Planung bisher noch keine Nerventransplantate notwendig, um eine spannungsfreie Koaptation $\mathrm{zu}$ ermöglichen. Es hat sich allerdings mit der Zeit eine Standardnerventransfermatrix sowohl für die transhumerale als auch für die glenohumerale Amputation durchgesetzt (- Tab. 1 und 2).

Auf glenohumeraler Ebene werden nach entsprechender Neurolyse des infraklavikulären Plexus aus der Stumpfnarbe die einzelnen Plexusanteile anhand der Medianuszinke identifiziert. Zielmuskeln auf dieser Amputationshöhe sind meist die drei Anteile des M. pectoralis major (Pars clavicularis, Pars sternocostalis, Pars abdominalis), der M. pectoralis minor sowie der M. latissimus dorsi und eventuell der M. infraspinatus. Es werden die segmentalen Nervi pectorales dargestellt und als Zielnerven angeschlungen [4]. Um auch hier eine größtmögliche räumliche Trennung der Muskelsignale zu erreichen, wird der M. pectoralis minor, gestielt an seinem Ast der A. thoracoacromialis, nach lateral verlagert. 
Unabhängig von der Amputationshöhe stimuliert man nach Darstellung aller Spender- und Zielnerven die einzelnen Äste und neurotomiert man eventuelle kleine interferierende Äste. Am Ende der Präparation muss jeder Zielmuskel selektiv ausschließlich über seinen Muskelast stimulierbar sein. Die einzelnen Nerven werden anschließend auf entsprechender Höhe neurotomiert, wobei dies möglichst muskelnah durchgeführt wird, um die Regenerationszeit und somit auch die Denervationszeit des Zielmuskels möglichst kurz zu halten. Alle Nerventransfers werden im Sinne einer Endzu-End-Koaptation unter Lupenvergrößerung oder dem OP-Mikroskop je nach Nervenkaliber mit 7-0- bis 10-0-monofiler Nylonnaht durchgeführt.

Um die Signalqualität und vor allem die Isolierung noch weiter zu optimieren, wird zuletzt die Haut, welche die Zielmuskeln überlagert, radikal entfettet [7] und bei der transhumeralen Amputationshöhe ein subkutaner Fettfaszienlappen zwischen kurzem und langem Kopf des M. biceps eingebracht.

\section{Nachbehandlung}

Die Nachbehandlung von TMR-Patienten ist mindestens so entscheidend wie die Operation selbst. Der Patient muss unter gezielter Anleitung eines/ einer Therapeuten/-in die Ansteuerung der einzelnen Muskelsignale erlernen. Dies wird mittels verschiedener technischer Möglichkeiten, wie z. B. EMG-Biofeedbacksysteme, unterstützt [18]. Hier können dem Patienten die einzelnen Signale visualisiert und mittels speziellen kognitiven Trainings an der Signaltrennung gearbeitet werden. Meist ist es für die Patienten zu Beginn einfacher, die einzelnen Bewegungen gleichzeitig mit der Gegenseite durchzuführen. Dies gibt auch dem Therapeuten eine Rückmeldung, an welche Bewegung gerade gedacht wird. Das intensive Signaltraining beginnt etwa drei bis sechs Monate nach erfolgter TMR-Operation, da etwa nach drei Monaten die ersten Muskelsignale aktiv werden. In den meisten Patienten ist die Nervenregeneration nach neun Monaten abgeschlossen, jedoch können sich auch zu diesem Zeitpunkt die

Unfallchirurg 2016 119:408-413 DOI 10.1007/s00113-016-0174-4

(c) The Author(s) 2016 . This article is available at SpringerLink with Open Access

\section{S. Salminger · J. A. Mayer · A. Sturma · O. Riedl · K. D. Bergmeister · O. C. Aszmann Prothetische Rekonstruktion der oberen Extremität}

\section{Zusammenfassung}

Hintergrund. Der prothetische Ersatz bei

Amputation oder Funktionsverlust der oberen Extremität hat in den letzten Jahren einen immer höheren und besonderen Stellenwert erlangt. Die Steuerung myoelektrischer Armprothesen konnte mittels Einsatz von selektiven Nerventransfers im Stumpfbereich deutlich verbessert sowie die Indikation zum prothetischen Ersatz erweitert werden.

Fragestellung. Übersicht über die chirurgischen, therapeutischen und prothetischen Möglichkeiten bei hohen Amputationen oder Funktionsverlust der oberen Extremität.

Material und Methode. Es erfolgte eine selektive Literaturrecherche unter Berücksichtigung eigener Erfahrungen des klinischen Alltags und Durchsicht von Patientenakten. Ergebnisse. Selektive Nerventransfers der amputierten Armnerven des Plexus brachialis auf verbliebene Stumpfmuskulatur können bis zu sechs Signalgeber schaffen, welche intuitiv und simultan die verschiedenen prothetischen Gelenke steuern können. Auf diese Weise ist eine effiziente und harmonische Steuerung der Prothese gewährleistet, ohne dass der Patient zwischen den verschiedenen Steuerungsebenen wechseln muss. Der prothetische Ersatz mit notwendiger elektiver Amputation stellt einen neuen Ansatz der funktionellen Extremitätenrekonstruktion bei Patienten mit funktionsloser Hand aufgrund massiver Weichteil- oder Nervenverletzung dar.

\section{Schlüsselwörter}

Amputationsstümpfe $\cdot$ Amputation . Prothesen · Nerventransfer · Plexus brachialis

\section{Prosthetic reconstruction of the upper extremity}

\section{Abstract}

Background. Prosthetic replacement after amputation or loss of function of the upper extremity has gained therapeutic value over the last years. The control of upper arm prostheses has been refined by the use of selective nerve transfers, and the indication for prosthetic replacement has been expanded.

Objectives. Overview regarding surgical, therapeutic and prosthetic options in upper extremity amputations or their loss of function.

Methods. Selective literature research including the authors' own experience in everyday clinical practice, as well as a review of medical records.

Results. Selective nerve transfers of the amputated nerves of the brachial plexus to the remaining stump muscles can create up to six myosignals for intuitive and simultaneous control of the different prosthetic joints. This way, an efficient and harmonious control of the prosthetic device is possible without the need to change between the different control levels. The prosthetic replacement, with consequent elective amputation, represents a new approach in the functional reconstruction of the upper extremity, especially in patients with a functionless hand after massive soft tissue or nerve damage.

\section{Keywords}

Amputation stumps · Amputation · Artificial limbs - Nerve transfer - Brachial plexus
Orte der besten Elektrodenpositionen noch ändern und somit eine erneute Schaftkorrektur notwendig machen.

\section{》) Die Technik eignet sich auch als Behandlungsmethode bei Phantomschmerzen}

Bei transhumeral Amputierten bleibt jeweils ein Teil des M. biceps und M. tri- ceps mit seiner ursprünglichen Innervation erhalten, wodurch die prothetische Versorgung mit konventioneller 2-Signalsteuerung nach Abschwellung des Stumpfes sofort wieder möglich ist. Bei glenohumeral Amputierten werden jedoch alle oberflächlich liegenden Muskeln im Stumpfbereich denerviert und somit ist die Steuerung einer myoelektrischen Prothese erst nach Reinnervation der ersten Muskelsignale möglich. 
Die Technik der TMR-Operation hat sich mittlerweile auch als solide Methode zur Behandlung von Neurom- bzw. Phantomschmerzen etabliert [17]. Denn schon ein einzelnes hypersensibles Neurom am Amputationsstumpf kann das Tragen einer Prothese unmöglich machen [2]. Die End-zu-End-Nervenkoaptation mit einem distalen Zielorgan ist die einzige bekannte Behandlungsmöglichkeit eines Neuroms, welche das grundlegendste Bedürfnis eines Nerven respektiert: das Vorhandensein eines funktionellen Endorgans [15]. Selektive Nerventransfers, wie bei einer TMR-Operation durchgeführt, stellen wieder eine sinnvolle Verbindung des betroffenen Nerven zu einem Endorgan her. Dadurch erhält das zentrale Nervensystem wieder eine brauchbare Rückmeldung, die schmerzhafte Lücke schließt sich und es kommt zu keiner erneuten Neurombildung.

Phantomschmerzen sind im Gegensatz zu den Neuromschmerzen deutlich schwieriger $\mathrm{zu}$ verstehen und auch $\mathrm{zu}$ behandeln. Als Hauptauslöser wird das Zentralnervensystem angenommen, welches aufgrund der fehlenden Afferenz ein meist schmerzhaftes Phantom der verlorenen Extremität kreiert [6]. Deshalb stellt der Phantomschmerz primär keine chirurgische Indikation dar, ist allerdings auch konservativ kaum behandelbar. Die klinische Erfahrung mit diesem Patientengut hat allerdings gezeigt, dass die durch selektive Nerventransfers neu geschaffenen Efferenzen und Afferenzen, in Kombination mit der prothetischen Versorgung, zu einer deutlichen Schmerzreduktion führen. Dem Gehirn wird, ähnlich dem Prinzip der Spiegeltherapie, durch die Prothese eine sensorische wie auch visuelle Rückmeldung geboten und somit eine funktionstüchtige Extremität simuliert.

\section{Fehler und Gefahren}

Da bei einer Amputationsverletzung das Endorgan der Spendernerven (N. medianus, N. ulnaris, N. radialis bzw. dessen R. profundus und N. musculocutaneus) fehlt, sind diese nicht stimulierbar. Somit ist die Identifizierung lediglich durch profunde anatomische Kenntnis möglich sowie die Funktionstüchtigkeit der Nerven nur klinisch, makroskopisch bzw. haptisch zu beurteilen. Dies ist vor allem bei eventuellen Traktionsschäden entscheidend, da ein proximaler Nervenschaden ausgeschlossen und der Spendernerv auf Höhe makroskopisch vitaler Faszikel rückgekürzt werden muss. Deshalb sollte die Technik der TMR-Operation nervenchirurgisch versierten Ärzten/innen vorbehalten bleiben, welche auch regelmäßig am Plexus brachialis operieren.

Weiters ist die Bedeutung der physiotherapeutischen und orthopädietechnischen Nachbetreuung nicht zu unterschätzen. Sie macht einen Großteil des funktionellen Outcomes aus und kann, bei mangelnder Betreuung des Patienten, eine gute Operation zunichtemachen.

\section{Die funktionslose Hand}

Manche schwerwiegenden Verletzungen der oberen Extremität führen zwar nicht notwendigerweise zu einer Amputation der Hand oder des Armes, ziehen aber einen mehr oder weniger großen Funktionsverlust nach sich. Dies gilt z. B. für massive Verletzungen des Plexus brachialis, aber auch für elektrische Verbrennungs-, Explosions- oder Avulsionstraumen, um nur ein paar wesentliche Verletzungsmuster zu nennen [3]. Auch in diesen Fällen hat die prothetische Rekonstruktion in den letzten Jahren einen immer höheren Stellenwert erlangt [3]. Keinesfalls sollen und können prothetische Möglichkeiten mit einer suffizienten biologischen Rekonstruktion konkurrieren, jedoch sollen sie das Spektrum erweitern und am Ende der biologisch rekonstruktiven Leiter eine sinnvolle Alternative bzw. Erweiterung zur Wiederherstellung einer Extremitätenfunktion bieten [3]. In diesen Fällen dient die Prothese als Rekonstruktion einer Hilfshand bzw. eines Hilfsarms, um den Patienten im täglichen Leben und bei der Arbeit zu unterstützen.

Eine austherapierte, nichtfunktionstüchtige Extremität mit einem Stück Technik zu ersetzen, stellt einen neuen Ansatz in der Extremitätenrekonstruktion dar, jedoch auch die Verschmelzung von Biologie und Technik, eine „bionische Rekonstruktion“ mit notwendiger elektiver Amputation der Hand oder Teilen davon unterliegt dem Grundsatz Primum non nocere. Somit stellt die adäquate Indikationsstellung sicherlich die größte Herausforderung dar.

\section{》) Eine bionische Rekonstruk- tion unterliegt dem Grundsatz Primum non nocere}

Im Folgenden wird anhand eines Patientenbeispiels der Prozess der prothetischen Rekonstruktion bei funktionsloser Extremität dargestellt.

Es handelt sich um einen 27 Jahre alten Mann, welcher im Rahmen eines Arbeitsunfalles in eine Walzmaschine gekommen ist, wodurch es zu einer Ablederung des gesamten Haut-WeichteilInteguments, von der Axilla bis zu den Fingerspitzen, des adominanten Armes gekommen ist. Es wurde versucht, die Extremität zu erhalten und den avulsierten Hautmantel im Sinne einer Vollhauttransplantation zur Deckung zu verwenden.

Aufgrund mangelnder Weichteildeckung kam es zu rezidivierenden Infekten und der Notwendigkeit multipler Fingerstrahl- bzw. Endgliedamputationen. Bis auf kleinste Wackelbewegungen war die linke Hand funktionslos und aufgrund der mangelnden Innervation, dystroph und asensibel. Ein prothetischer Aufbau auf die vorhandenen Fingerstummel bzw. Teilhand wurde versucht, war allerdings aufgrund der schlechten Hautqualität bzw. des eingeschränkten Bewegungsausmaßes erfolglos (• Abb. 1).

Daraufhin wurde der Patient mit einer sog. Hybridprothese provisorisch versorgt. Hier wird eine myoelektrische Prothese mithilfe einer Schienenkonstruktion unter der existierenden Hand montiert, um die vorhandenen Myosignale $\mathrm{zu}$ testen und $\mathrm{zu}$ trainieren sowie dem Patienten die zu erwartende prothetische Funktion vor Augen zu führen (- Abb. 2). Dies stellt einen wichtigen Schritt in der Entscheidungsfindung sowohl für den Patienten als auch für den Arzt dar. Erst wenn der Patient mit dieser Versorgung einen deutlichen Funktionsgewinn wahrnehmen kann und dieser auch objektiv in Handfunktionstests be- 


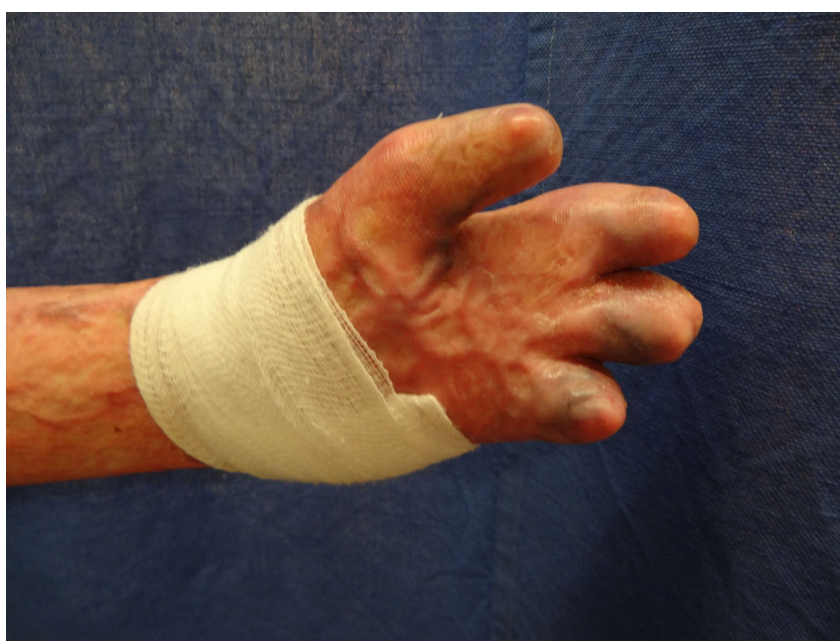

Abb. $1 \Delta$ Funktionslose Hand nach massiver Weichteilverletzung

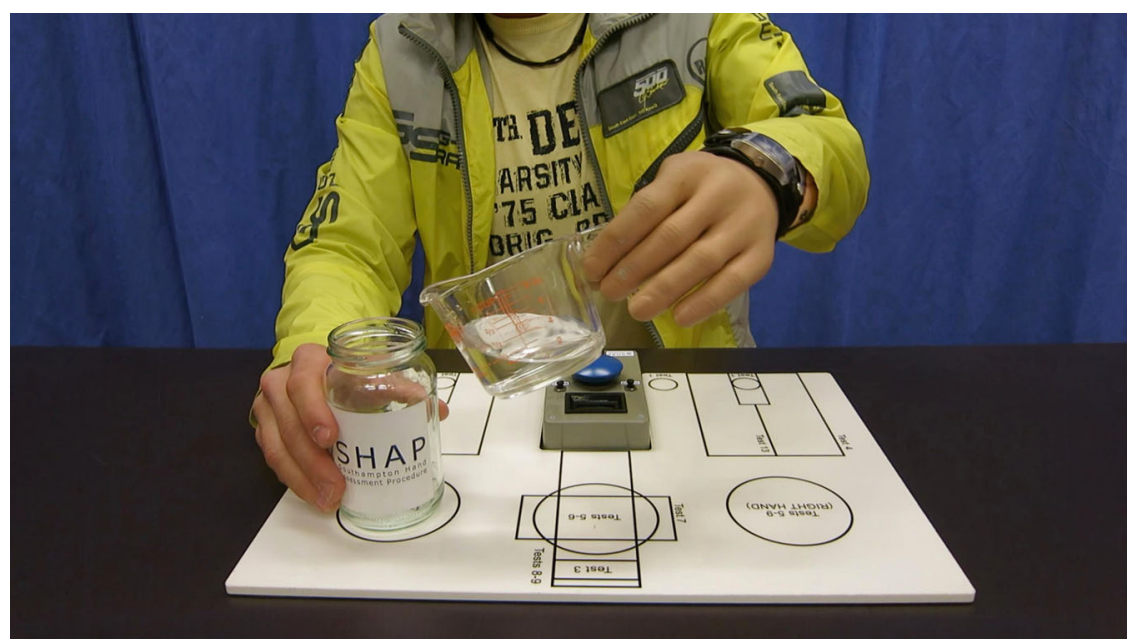

Abb. $3 \Delta$ Funktionstests nach elektiver Amputation und Prothesenversorgung

legbar ist (s. Video online), kann eine elektive Amputation, auf Wunsch des Patienten, geplant werden. Weiters wird der Patient präoperativ einer psychologischen Evaluation unterzogen, um Copingmechanismen entsprechend abzuklären.

Aufgrund der beeindruckenden Handfunktion dieser Hybridprothese hat sich der Patient für eine transkarpale Amputation entschieden, um Platz für eine prothetische Handfunktion $\mathrm{zu}$ schaffen und eine suffiziente, ausreichend sensible Weichteildeckung $\mathrm{zu}$ gewährleisten. In etwa 6 Wochen nach Amputation war der Stumpf ausreichend abgeschwollen, die Wunden waren gut verheilt und es konnte mir der prothetischen Versorgung begonnen werden. Kurze Zeit nach der Prothesenanpas-

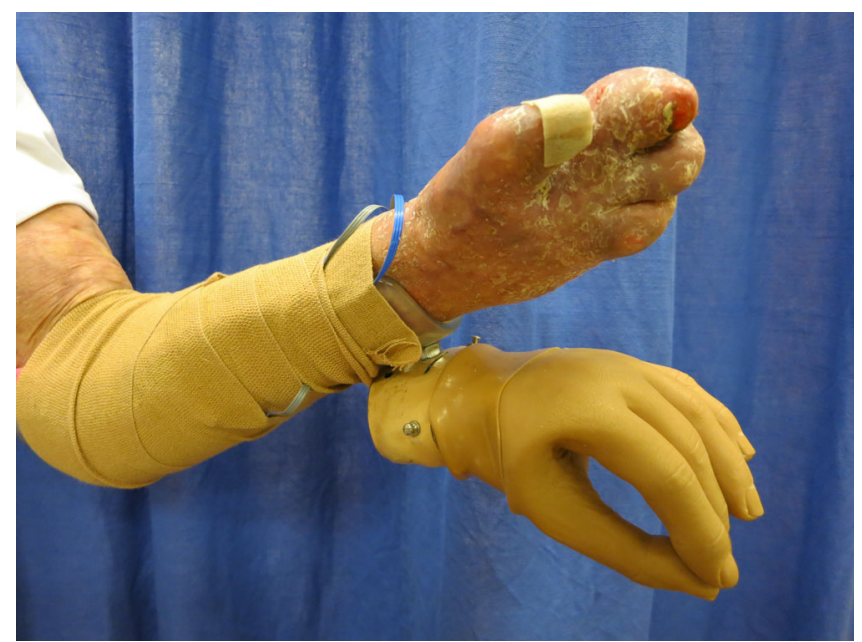

Abb. 2 ム Hybridprothesenversorgung

\section{Zukünftige Weiter- entwicklungen}

Zum jetzigen Zeitpunkt werden die Muskelsignale mittels Oberflächenelektroden aufgenommen und an die Prothese weitergeleitet. Aufgrund von Weichteilverschiebungen über einem Muskel, der variablen Elektroden- bzw. Stumpfposition im Schaft, Kontaktverlust durch Hebelwirkungen und der Impedanzschwankungen je nach Temperatur und Transpiration der Haut kann es zu Fehlschaltungen kommen, welche die Prothesenfunktion negativ beeinflussen. Durch intramuskulär implantierte Elektroden kann das Signal direkt aus dem Muskel an die Prothese weitergeleitet und somit auch die Signalqualität deutlich verbessert werden [12].

Unabhängig von der Signalextraktion wird die Verarbeitung der verschiedenen Muskelsignale mittels Mustererkennung eine noch intuitivere Prothesensteuerung ermöglichen, welche zusätzlich durch simple Feedbackmechanismen verfeinert werden wird $[5,13]$.

\section{Diskussion}

Funktionsverlust oder Amputationen der oberen Extremität stellen für betroffene Patienten einschneidende und traumatische Erlebnisse dar, welche oft mit massiven Einbußen in der Lebensqualität einhergehen [10]. Der prothetische Ersatz, auch wenn in manchen Fällen mit einer notwendigen Amputation einher- 
gehend, solch funktioneller Defizite ermöglicht eine Reintegration ins Arbeitsund Sozialleben und somit eine verbesserte Lebensqualität.

\section{》) Die TMR-Operation ermöglicht eine verbesserte Prothesensteuerung}

Die TMR-Operation ermöglicht Patienten mit hohen Amputationen der oberen Extremität eine deutlich verbesserte und vor allem intuitive Prothesensteuerung und behandelt gleichzeitig vorhandene Neurome am Amputationsstumpf. Die Möglichkeit der simultanen Ansteuerung der einzelnen prothetischen Gelenke beschleunigt die Durchführung verschiedenster Tätigkeiten, bei denen die Prothese als Hilfshand dienen kann, sowohl im Privat- als auch im Berufsleben.

Der prothetische Extremitätenersatz bei funktionsloser Hand stellt einen neuen Ansatz der funktionellen Wiederherstellung dar, welcher vor allem für $\mathrm{Pa}$ tienten infrage kommt, bei denen alle Möglichkeiten der biologischen Rekonstruktion ausgeschöpft sind und trotzdem eine funktionslose, asensible und somit unbrauchbare Hand besteht. In diesem Konzept spielt die Hybridversorgung, wie oben beschrieben, eine entscheidende Rolle und dient als zentrales Instrument in der Entscheidungsfindung, sowohl für den Patienten als auch für das behandelnde Team.

\section{Fazit für die Praxis}

Die dargestellten Konzepte des prothetischen Extremitätenersatzes erfordern eine funktionierende interdisziplinäre Zusammenarbeit verschiedenster Disziplinen wie Chirurgie, Physiotherapie und physikalische Medizin, Techniker und Orthopädietechniker, aber auch Psychologen und Wissenschaftler. An der Medizinischen Universität Wien ist hierfür ein eigenes Zentrum (Christian Doppler Labor für Wiederherstellung von Extremitätenfunktionen) gegründet worden, welches aus einem ca. 15-köpfigen Team besteht, um diese aufwendigen Therapien anbieten zu können. Somit sollte die komplexe Re- konstruktion mit oben vorgestellten Methoden von Zentren durchgeführt werden, die über solch personelle aber auch infrastrukturelle Ressourcen verfügen.

\section{Korrespondenzadresse}

Univ.-Prof. Dr. O. C. Aszmann

Klinische Abteilung für Plastische und Rekonstruktive Chirurgie, Universitätsklinik für Chirurgie, Medizinische Universität Wien Währinger Gürtel 18-20, 1090 Wien, Österreich oskar.aszmann@meduniwien.ac.at

\section{Einhaltung ethischer Richtlinien}

Interessenkonflikt. S. Salminger, J. A. Mayer, A. Sturma, O. Riedl, K. D. Bergmeister und O. C. Aszmann geben an, dass kein Interessenkonflikt besteht.

Dieser Beitrag beinhaltet keine von den Autoren durchgeführten Studien an Menschen oder Tieren.

Alle Patienten, die über Bildmaterial oder anderweitige Angaben innerhalb des Manuskripts zu identifizieren sind, haben hierzu ihre schriftliche Einwilligung gegeben.

Open Access. This article is distributed under the terms of the Creative Commons Attribution 4.0 International License (http://creativecommons.org/ licenses/by/4.0/), which permits unrestricted use, distribution, and reproduction in any medium, provided you give appropriate credit to the original author(s) and the source, provide a link to the Creative Commons license, and indicate if changes were made.

\section{Literatur}

1. Aszmann OC et al (2008) Selective nerve transfers to improve the control of myoelectrical arm prostheses. Handchir Mikrochir Plast Chir 1(40):60-65

2. Aszmann OC et al (2010) Treatment of painful neuromas via end-to-side neurorraphy. Handchir Mikrochir Plast Chir 4(42):225-232

3. Aszmann OC et al (2015) Bionic reconstruction to restore hand function after brachial plexus injury: a case series of three patients. Lancet 385:2183

4. Aszmann OC et al (2000) The anatomy of the pectoral nerves and their significance in brachial plexus reconstruction. J Hand Surg Am 25(5):942-947

5. Farina D et al (2014) Noninvasive, accurate assessment of the behavior of representative populations of motor units in targeted reinnervated muscles. IEEE Trans Neural Syst Rehabil Eng 22(4):810-819

6. Flor Het al (1995) Phantom-limb pain as a perceptual correlate of cortical reorganization following arm amputation. Nature 375(6531):482-484

7. Kuiken TA et al (2003) The effect of subcutaneous fat on myoelectric signal amplitude and cross-talk. Prosthet Orthot Int 27(1):48-54

8. Kuiken TA et al (2004) The use of targeted muscle reinnervation for improved myoelectric prosthesis control in a bilateral shoulder disarticulation amputee. Prosthet Orthot Int 28(3):245-253

9. Leixnering $M$ et al (2013) Prevention of hand injuries - current situation in Europe. Handchir Mikrochir Plast Chir 6(45):339-343

10. Meyer TM (2003) Psychological aspects of mutilating hand injuries. Hand Clin 19(1):41-49

11. Oleske DM, Hahn JJ (1992) Work-related injuries of the hand: data from an occupational injury/ illness surveillance system. J Community Health 17(4):205-219

12. Pasquina PF et al (2014) First-in-man demonstration of a fully implanted myoelectric sensors system to control an advanced electromechanical prosthetic hand. J Neurosci Methods. doi:10.1016/j.jneumeth.2014.07.016

13. Roche AD et al (2014) Prosthetic Myoelectric Control Strategies: A Clinical Perspective. Curr Surg Reports 2(3):44

14. Salminger $S$ et al (2015) Prosthetic reconstruction in high amputations of the upper extremity. Orthopade 44:413

15. Salminger S et al (2014) Immediate and secondary reconstruction of iatrogenic nerve lesions. Chir Prax 78:625-637

16. Schroeder HPVon, BotteMJ(1998)Crush syndrome of the upper extremity. Hand Clin 14(3):451-456

17. Souza JM et al (2014) Targeted muscle reinnervation: A novel approach to postamputation neuroma pain. Clin Orthop Relat Res 472:2984

18. Sturma A et al (2015) A surface EMG test tool to measure proportional prosthetic control. Biomed Tech (Berl) 60(3):207-213 